\title{
The LOFT contribution to GRB science
}

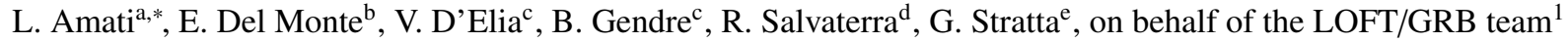 \\ ${ }^{a}$ INAF - IASF Bologna, via P. Gobetti 101, 40129 Bologna, Italy \\ ${ }^{b}$ INAF - IAPS, via Fosso del Cavaliere 100, 00133 Roma, Italy \\ ${ }^{c}$ ASI Science Data Center (ASDC), via Galileo Galilei, 00044 Frascati, Italy \\ ${ }^{d}$ INAF - IASF Milano, via E. Bassini 15, I-20133 Milano, Italy \\ ${ }^{e}$ INAF - Osservatorio Astronomico di Roma, via Frascati 33, 00040 Monte Porzio Catone (RM), Italy
}

\begin{abstract}
LOFT is a satellite mission currently in Assessment Phase for the ESA M3 selection. The payload is composed of the Large Area Detector (LAD), with 2-50 keV energy band, a peak effective area of about $10 \mathrm{~m}^{2}$ and an energy resolution better than $260 \mathrm{eV}$, and the Wide Field Monitor (WFM), a coded mask imager with a FOV of several steradians, an energy resolution of about $300 \mathrm{eV}$ and a point source location accuracy of 1 arcmin in the $2-30 \mathrm{keV}$ energy range. Based on preliminary considerations and estimates, we show how the scientific performances of the WFM are particularly suited to investigate some of the most relevant open issues in the study of GRBs: the physics of the prompt emission, the spectral absorption features by circum-burst material (and hence the nature of the progenitors), the population and properties of XRFs, and the detection and rate of high-z GRBs. Measurements of the early afterglow emission with the Large Area Detector (LAD) may also be possible depending on the mission slewing capabilities and TOO observations policy.
\end{abstract}

Keywords: $\mathrm{X}$-ray astronomy: instrumentation, gamma-rays: bursts

\section{Introduction}

Despite of huge advances occurred in the last 10 years, the Gamma-ray Bursts (GRB) phenomenon is still far to be fully understood. Open issues include: physics and geometry of the prompt emission, unexpected early afterglow phenomenology (e.g. plateau phase, flares), identification and understanding of subclasses of GRBs (short/long, X-Ray Flashes, subenergetic GRBs), GRB/SN connection, nature of the inner engine, cosmological use of GRBs, and more. See, e.g., [1, 2, 3] for recent reviews.

The Large Observatory For X-ray Timing, LOFT [4, 5], was selected in 2011 by the European Space Agency as one of the four Cosmic Vision M3 candidate missions to compete for a launch opportunity at the start of the

\footnotetext{
*Email: amati@iasfbo.inaf.it

${ }^{1}$ http://www.iasfbo.inaf.it/ amati/loftgrb
}

2020s. The current schedule of the ESA/M3 programme foresees the end of the assessment study by the end of 2013 and the final down-selection in the first months of 2014.

Thanks to an innovative design and the development of large-area monolithic silicon drift detectors, the Large Area Detector (LAD) on board LOFT will operate in the $2-30 \mathrm{keV}$ range (up to $80 \mathrm{keV}$ in expanded mode) with a FOV collimated to $\sim 1^{\circ}$, and achieve an effective area of $\sim 10 \mathrm{~m}^{2}$ at $8 \mathrm{keV}$, a time resolution of $\sim 10$ $\mu$ s and a spectral resolution of $\sim 200-260 \mathrm{eV}$ (FWHM at $6 \mathrm{keV}$ [6, 7]. These capabilities will allow LOFT to investigate with unprecedented sensitivity the rapid Xray flux and spectral variability that directly probes the motion of matter down to distances very close to black holes and neutron stars, as well as the physical state of ultra-dense matter [4]. Such measurements are efficient diagnostics of the behavior of matter in the presence of strong gravitational fields, where the effects predicted 
Table 1: Main characteristics of the LOFT Wide Field Monitor (WFM) [8]. 5 .

\begin{tabular}{|c|c|c|}
\hline Parameter & Requirement & Goal \\
\hline $\begin{array}{l}\text { Point Source } \\
\text { Location Accuracy }\end{array}$ & $<1$ arcmin & $<0.5$ arcmin \\
\hline Angular Resolution & $<5$ arcmin & $<3$ arcmin \\
\hline $\begin{array}{l}\text { Sensitivity } \\
(5 \sigma, \text { on-axis })\end{array}$ & $\begin{aligned} & 1 \mathrm{~s}: 1 \mathrm{Crab} \\
& 3 \times 10^{-8} \mathrm{erg} / \mathrm{cm}^{2} / \mathrm{s} \\
& 50 \mathrm{ks}: 5 \mathrm{mCrab} \\
& 1.5 \times 10^{-10} \\
& \mathrm{erg} / \mathrm{cm}^{2} / \mathrm{s}\end{aligned}$ & $\begin{aligned} 1 \mathrm{~s}: & 0.2 \mathrm{Crab} \\
& 6 \times 10^{-9} \mathrm{erg} / \mathrm{cm}^{2} / \mathrm{s} \\
50 \mathrm{ks}: & 2 \mathrm{mCrab} \\
6 & \times 10^{-11} \mathrm{erg} / \mathrm{cm}^{2} / \mathrm{s}\end{aligned}$ \\
\hline Field of View & $\begin{array}{l}\pi \text { sr around the LAD } \\
\text { pointing }\end{array}$ & $\begin{array}{l}1.5 \pi \mathrm{sr} \text { around the LAD } \\
\text { pointing }\end{array}$ \\
\hline Energy Range & $\begin{array}{l}2-30 \mathrm{keV} \text { (primary); } \\
30-80 \mathrm{keV} \text { (extended) }\end{array}$ & $\begin{array}{l}1.5-30 \mathrm{keV} \text { (primary); } \\
30-80 \mathrm{keV} \text { (extended) }\end{array}$ \\
\hline Energy Resolution & $500 \mathrm{eV}$ & $300 \mathrm{eV}$ \\
\hline
\end{tabular}

by General Relativity are more relevant, and the physics of matter at densities in excess of that in atomic nuclei, determining its equation of state and composition.

In addition to the LAD, the LOFT payload will include a Wide Field Monitor (WFM) [8], which will monitor more than half of the LAD-accessible sky (approximately $1 / 3$ of the whole sky) simultaneously at any time, will operate in the same energy range as the LAD with an energy resolution of $\sim 300 \mathrm{eV}$, providing information about source status (flux variability and energy spectrum), as well as arc-minute positioning (Tab. 1). With such wide angle sky monitoring, the WFM will also provide long term histories of the target sources, thus facilitating both the LAD observations and a series of wider science goals.

Based on preliminary considerations and estimates, we show that LOFT, possibly in combination with other GRB experiments flying at the same epoch, will give us useful and unique clues to some of these still open issues in this field, through: a) measurements of the prompt emission down to $\sim 2 \mathrm{keV}$ and $\sim$ arcmin localization with the Wide Field Monitor (WFM); b) measurements of the early afterglow emission with the Large Area Detector (LAD).

As detailed below, the partly unprecedented characterization of the GRB X-ray prompt emission, joined with source location accurate enough for optical followup, by the WFM will allow to investigate the properties of the circum-burst environment, thus getting further clues on the nature of the progenitors, to provide a more stringent tests for the emission mechanisms at play, to increase the detection rate of high-z GRBs w/r to previous missions, to shade light on the population of
X-ray Flashes (XRFs) and sub-energetic events. While the GRB science with the WFM will come 'for free', provided the optimization of trigger logic, data modes and prompt (within a few tens of s) transmission of trigger time and position to ground (which is presently in the baseline of the mission [5, 8] ), the contribution from the LAD, consisting in the possible further characterization of the 'plateau phase' of the early X-ray afterglow emission, will critically depend on the follow-up capabilities and policy of the mission.

\section{GRB science with the LOFT/WFM}

The WFM will achieve scientific goals of fundamental importance and not fulfilled by GRB experiments presently flying (e.g., Swift[9], Konus/WIND[10], Fermi/GBM[11]) and future approved missions (SVOM[12], UFFO[13]). These can be summarized as follows:

- measurement of the GRB spectral shape and its evolution down to about $2 \mathrm{keV}$ in photon energy which is crucial for testing models of GRB prompt emission (still to be settled despite the considerable amount of observations; see, e.g., [14]);

- detection and study of transient X-ray absorption column / features for a few bright GRBs per year (Fig. 1). These measurements are of paramount importance for the understanding of the properties of the Circum-Burst Matter (CBM) and hence the nature of GRB progenitors (still a fundamental open issue in the field). In addition, as demonstrated by BeppoSAX [15], the detection of tran- 


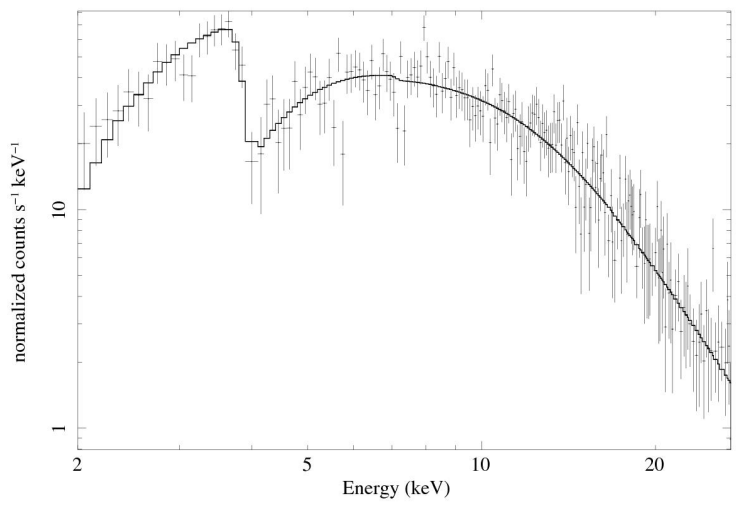

Figure 1: The transient absorption edge detected in the first $13 \mathrm{~s}$ of GRB 990705 [15] as would be measured by the LOFT/WFM.

sient X-ray spectral features can allow the determination of the GRB redshift to be compared, when it is the case, with that determined from the optical/NIR lines;

- to provide a substantial increase (with respect to the past and current missions) in the detection rate of X-Ray Flashes (XRF), a sub-class of soft / ultra-soft events which could constitute the bulk of the GRB population (e.g., [16]) and still have to be explored satisfactorily;

- to extend the GRB detection up to very high redshift ( $z>8$ ) GRBs (Fig. 2), which is of fundamental importance for the study of evolutionary effects, the tracing of star formation rate, ISM evolution, and possible unveiling of population III stars (e.g., [17, 18]);

- to provide fast $(<30 \mathrm{~s})$ and accurate $(\sim 1$ arcmin $)$ location of the detected GRBs to allow their prompt multi-wavelength follow-up with ground and space telescopes, thus leading to the identification of the optical counterparts and/or host galaxies and to estimate the redshift, a fundamental measurement for the scientific goals listed above, comparing it with that determined from X-ray absorption lines.

\section{Possible further contribution by the LOFT/LAD}

The GRB science that could be done with the LAD is less 'automatic' and is strongly dependent on the time

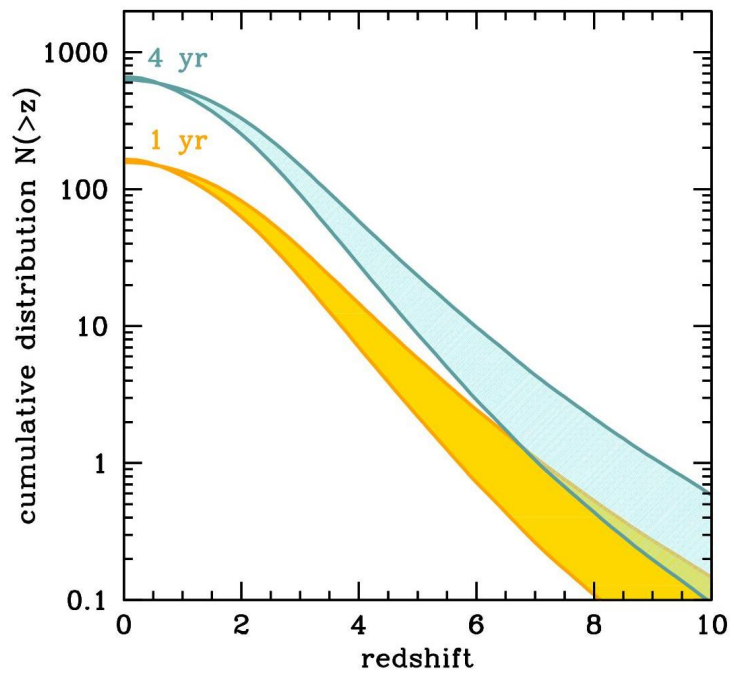

Figure 2: Expected GRB +XRF detection rate by the LOFT/WFM as a function of redshift. The width of the strips reflect the uncertainties in the GRB luminosity function and its evolution with redshift (see [17]).

that will be required to start a TOO observation. Possible GRB science that could be performed by the LAD include:

- investigating the plateau phase of the early X-ray afterglow and its transition to the 'normal decay' (Fig. 3), which is of high importance e.g. for the search of signatures of energy injection by a magnetized NS and to better investigate the correlation between time and luminosity of the transition from plateau to normal decay (e.g., [19]);

- searching for emission lines, expected by theoretical models in case of highly metal enriched circum-burst environment and detected in a few cases by BeppoSAX, Chandra and XMM (e.g., [20]) but not by Swift/XRT;

- complementing the observations of the prompt emission by the WFM by exploiting the transparency of the collimator at energies $>30-40 \mathrm{keV}$.

\section{LOFT GRB science in the $>2020$ context}

No past, present or future (e.g., SVOM, UFFO) GRB experiment has such a combination of low energy threshold and high energy resolution (and wide FOV), which will make the LOFT/WFM unique also for GRB science. For instance, the BeppoSAX/WFC or the HETE-2/WXM had a low energy threshold around 2 

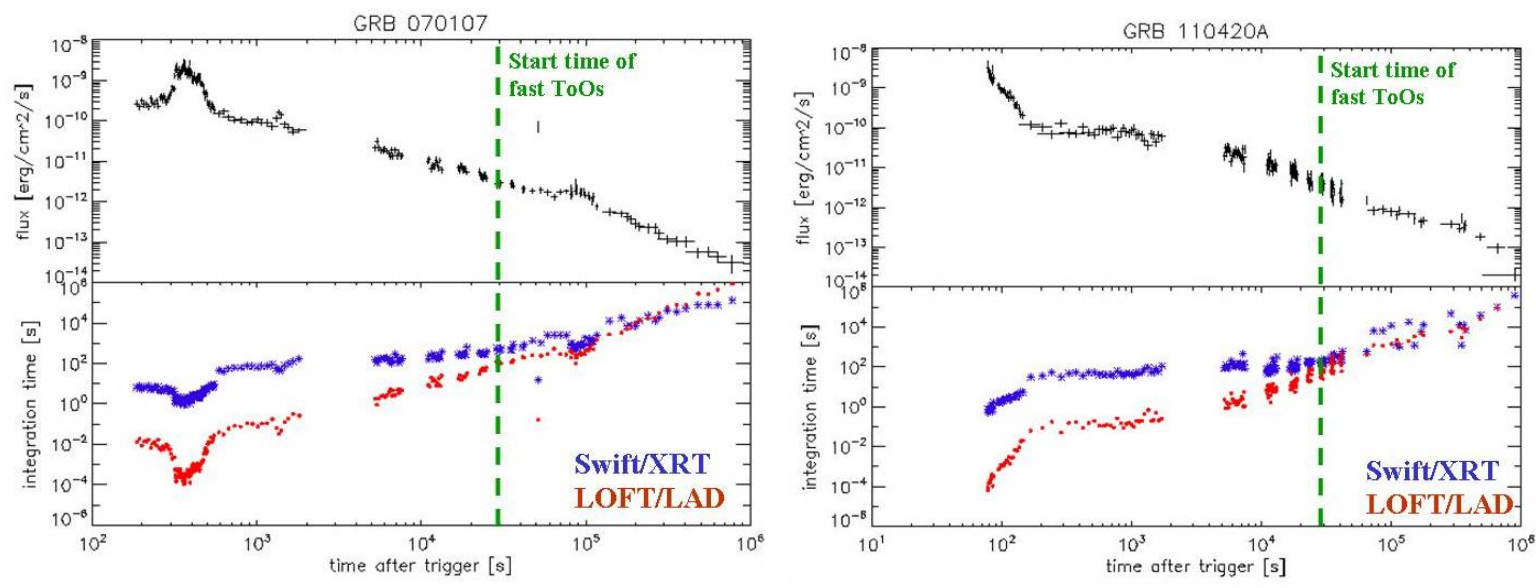

Figure 3: Top panels: light curves of the early afterglow of two GRBs observed by Swift. Bottom panels: time required to reach a $5 \sigma$ significance in a given time bin for the Swift/XRT and the LOFT/LAD. As can be seen, the LOFT/LAD would be more sensitive than the Swift/XRT up to about 8 hours from the GRB onset.

$\mathrm{keV}$, but with much worse energy resolution and smaller effective area (and smaller FOV). SVOM and UFFO (>2018) will have a low energy threshold of $\sim 5 \mathrm{keV}$ and with significantly worse effective area and energy resolution $\mathrm{w} / \mathrm{r}$ to LOFT/WFM at energies $<10 \mathrm{keV}$. In addition, in particular thanks to the capability of promptly transmitting the GRB trigger and a position with a few arcmin uncertainty [8], LOFT will: a) continue the fundamental 'service' to the astrophysical community, carried out presently by Swift (and possibly in the 20182022 time frame by SVOM and/or UFFO) of providing detection, prompt localization and temporal / spectral characterization of GRBs, thus allowing their followup and multi-wavelength studies with the best telescopes operating in the $>2020$ time line (e.g., LSST, SKA, CTA, eROSITA, maybe XMM, Chandra, etc.); b) complement simultaneous observations by GRB experiments flying on other satellites by providing low energy extension and GRB position, in a way similar to what is presently done, e.g., by joining data from Swift, Fermi and Konus/WIND.

In addition, the study of GRBs, because of their complex phenomenology and extreme energetics, the association of at least a fraction of them with core-collapse $\mathrm{SNe}$, their redshift distribution extending up to at least $\mathrm{Z} \sim 8-9$, is relevant to different fields of astrophysics, ranging from plasma and black-hole physics to cosmology. In particular, the LOFT measurements summarized above will provide not only a step forward in the understanding of GRB physics, progenitors and sub-classes, but, also in combination with observations by the best instrumentation at all wavelengths, will shade light on fundamental topics like the evolution of the star form- ing rate and of the galactic ISM up to the epoch of reionization, the first generation (pop III) stars, the understanding of the diversity and rate of core-collapse SNe, the measurement of cosmological parameters.

Finally, the GRB science already involves, and will continue to involve at the time of LOFT, observers and instrumentation from different communities: optical/IR robotic telescopes (prompt detections and localization of the optical counterparts), major optical/IR telescopes like VLT, Gemini, Hubble (identification, redshift and ISM of the host galaxies, optical afterglow decay and jet signatures), radio telescopes like VLA (afterglow modeling and energetics) observations in the $\mathrm{TeV}$ range by Cherenkov telescopes (challenging emission models).

\section{References}

[1] P. Mészaros, Rep. Prog. Phys. 69 (2006) 2259.

[2] N. Gehrels, E. Ramirez-Ruiz, D.B. Fox, ARA\&A 47 (2009) 567.

[3] B. Zhang, Comptes Rendus Physique 12 (2011) 206 arXiv:1104.0932).

[4] M. Feroci, L. Stella, M. van der Klis, et al., Experimental Astronomy 34 (2012) 415.

[5] M. Feroci, J.W. den Herder, E. Bozzo, et al., Proceedings of the SPIE 8443 (2012) id.2.

[6] E. Bozzo, J.W. den Herder, M. Feroci, L. Stella, Proceedings of The Extreme and Variable High Energy Sky (Proceedings of Science: Extremesky 2011) (2011) id.25.

[7] S. Zane, D. Walton, T. Kennedy, et al., Proceedings of the SPIE 8443 (2012) id. 15.

[8] S. Brandt, M. Hernanz, L. Alvarez, et al., Proceedings of the SPIE 8443 (2012) id.14.

[9] N. Gehrels, G. Chincarini, P. Giommi, et al., ApJ 611 (2004) 1005.

[10] R.L. Aptekar, D.D. Frederiks, S.V. Golenetskii, et al., Space Science Reviews 71 (1995) 265. 
[11] C. Meegan, G. Lichti, P.N. Bhat, et al., ApJ 702 (2009) 791.

[12] O. Godet, J. Paul, J.Y. Wei, et al., Proceedings of the SPIE 8443 (2012) id.10.

[13] B. Grossan, I.H. Park, S. Ahmad, et al. ,Proceedings of the SPIE 8443 (2012) id.13.

[14] B. Zhang, P. Mészáros, ApJ 581 (2002) 1236.

[15] L. Amati, F. Frontera, M. Vietri, et al., Science 290 (2000) 953.

[16] A. Pelangeon, J.-L. Atteia, Y. E. Nakagawa, et al., A\&A 491 (2008) 157.

[17] R. Salvaterra, S. Campana, S. Vergani, G., et al., ApJ 749 (2010) 68.

[18] S.E. Woosley, J.S. Bloom, ARA\&A 44 (2006) 507.

[19] B. Zhang, Chinese Journal of Astronomy and Astrophysics 7 (2007) 1.

[20] L. Piro, G. Garmire, M. Garcia, et al., Science 290 (2000) 955. 